\title{
TERMS OF THE ARC AND SPARK SPECTRA OF CHROMIUM
}

\author{
By C. C. Kiess
}

ABSTRACT

Tables of terms are presented for the arc and first spark spectra of chromium. Babcock's unpublished Zeeman effects have aided in establishing many of them. Combinations of these terms account for nearly all the stronger lines of $\mathrm{Cr}$ I and Cr II. All the terms which have been identified conform to the requirements of Hund's theory.

\section{CONTENTS}

I. Introduction .... 775

II. Tables of terms of $\mathrm{Cr}$ I and $\mathrm{Cr}$ II

\section{INTRODUCTION}

The terms of Cr I and Cr II, presented herewith in Tables 1 and 2, are published at this time in response to numerous requests which have been made of the National Bureau of Standards for the classifications of the more prominent lines in the arc and spark spectra of chromium. The analyses of these spectra have been in progress at various times during the past decade at the bureau and elsewhere, and although the task is by no means completed, yet the terms already identified account for nearly all the strong lines of these spectra which are likely to be of interest to astronomers, physicists, and chemists.

The classifications made at the bureau have been greatly facilitated by new measurements of wave lengths in the arc and spark spectra, by King's temperature classifications, ${ }^{1}$ and by Babcock's unpublished Zeeman effects, kindly placed at our disposal by him for this work. The terms of $\mathrm{Cr}$ I (Table 1), belong to the septet, quintet, and triplet systems and include those already published. ${ }^{2}$ The terms of Cr II, and classifications based on them, belong to the sextet, quartet, and doublet systems. They have been partially described in previous communications and publications, ${ }^{3}$ from which it would be possible to derive nearly all that have been found thus far. But for convenience they are now brought together in Table 2. Many of them have been confirmed by Krömer from an investigation of the Zeeman effect of $\mathrm{Cr}^{+}$ at Tübingen. However, the term proposed by him for $z^{6} \mathrm{D}^{\circ}$ is undoubtedly erroneous; and the pair of lines at 3291.76 and $3369.06 \mathrm{~A}$,

1 Astrophys. J., 41, p. 167; 1915; 60, p. 282; 1924.

Kiess and Kiess, Science, 56, p. 666; 1922. Catalán, Phil. Trans. Roy. Soc., A 223, p. 163; 1922; Anales Soc. Españ. Fis. Quim., 21, p. 84; 1923. Gieseler, Ann. der Physik, 69, p. 147; 1922; Zeit. für Physik, 22, p. 228; 1924. Zumstein, Phys. Rev., 27, p. 565; 1926. St. John, Revision of Rowland's Preliminary Table, p. 228; 1928. Ruark and Urey, Atoms, Molecules and Quanta, pp. 302-312; McGraw-Hill, New York, 1930. Meggers, Kiess, and Walters, J. Opt. Soc. Am. and Rev. Sci. Inst., 9, p. 355; 1924. Kiess, J. Opt. Soc. Am. and Rev. Sci. Inst., 10, p. 287; 1925. Kiess and Laporte, Science, 63, p. 234; 1926. St. John, Revision of Rowland's Preliminary Table, p. 229; 1928. Dunham and Moore, Astrophys. J., 68, p. 37; $1928 . \quad$ Krömer, Zeit. für Physik, 52, p. 531; 1928, Dunham, Contrib, Princeton Univ, Observatory, No. 9; 1929. 
regarded by him as emitted by the trebly ionized $\mathrm{Cr}$ atom, are accounted for by the doublet terms given below. Quite recently Catalán ${ }^{4}$ has published a partial analysis of Cr II, his terms agreeing for the most part with those given below. The symbols used in designating the terms are those recently proposed as standard by Russell, Shenstone, and Turner. ${ }^{5}$

According to present theories of atomic structure the terms which characterize a spectrum represent the resultants of the interaction of the electrons outside the closed shells. Hund's theory ${ }^{6}$ specifies these terms as to number and type for any particular electron configuration. The low and metastable terms of Cr I result from the configurations $d^{5} s$ and $d_{s}^{4} s^{2}$. All the septet and quintet terms which arise from these electron groups have been found except ${ }^{5} \mathrm{~F}$. Of the triplet terms a few have been found but have not yet been connected with the quintets through intersystem combinations. No singlets have been established thus far. The configurations $d^{5}, d^{4} s$, and $d^{3} s^{2}$ give the low and metastable terms of Cr II. All the sextet and most of the quartet terms possible from these configurations are given in Table 2 . Of the doublet terms only a few have been found. The higher terms which arise in both spectra, when an electron is dislodged to a $p$ orbit, have been only incompletely identified. As stated above, however, the terms which have been established account for nearly all the stronger lines of the spectra and should serve to classify those lines of Cr I and Cr II which are likely to be of astrophysical and spectrochemical importance. Further discussion of the terms and their interpretation will be deferred until the complete classifications of the spectra are presented in subsequent papers.

\section{TABLES OF TERMS OF Cr I AND Cr II}

TABLE 1.-Terms of $\mathrm{Cr} I$

\begin{tabular}{|c|c|c|c|c|c|}
\hline Term & $\nu$ & $\Delta \nu$ & Term & $\nu$ & $\Delta \nu$ \\
\hline $\begin{array}{l}a^{7} \mathrm{~S}_{3} \\
e^{7} \mathrm{~S}_{3}\end{array}$ & $\begin{array}{r}0.0 \\
36,895.8\end{array}$ & & $\begin{array}{l}a^{5} \mathrm{~S}_{2} \\
e^{5} \mathrm{~S}_{2} \\
f^{5} \mathrm{~S}_{2}\end{array}$ & $\begin{array}{r}7,593.1 \\
37,883.1 \\
45,967.7\end{array}$ & \\
\hline $\begin{array}{l}e^{7} D_{5} \\
e^{7} D_{4} \\
e^{7} D_{3} \\
e^{7} D_{2} \\
e^{7} D_{1}\end{array}$ & $\begin{array}{l}42,261.3 \\
42,258.4 \\
42,256.2 \\
42,254.5 \\
42,253.3\end{array}$ & $\begin{array}{l}2.9 \\
2.2 \\
1.7 \\
1.2\end{array}$ & $\begin{array}{l}a^{5} \mathrm{P}_{3} \\
a^{5} \mathrm{P}_{2} \\
a^{5} \mathrm{P}_{1} \\
a^{5} \mathrm{D}_{4}\end{array}$ & $\begin{array}{l}21,840.7 \\
21,847.9 \\
21,857.0\end{array}$ & $\begin{array}{l}-7.2 \\
-9.1\end{array}$ \\
\hline $\begin{array}{l}f^{7} D_{5} \\
f^{7} D_{4} \\
f^{7} D_{3} \\
f^{7} D_{2} \\
f^{7} D_{1}\end{array}$ & $\begin{array}{l}46,959.1 \\
46,782.9 \\
46,637.2 \\
46,524.9 \\
46,448.6\end{array}$ & $\begin{array}{r}176.2 \\
145.7 \\
112.3 \\
76.3\end{array}$ & $\begin{array}{l}a^{5} \mathrm{D}_{4} \\
a^{5} \mathrm{D}_{3} \\
a^{5} \mathrm{D}_{2} \\
a^{5} \mathrm{D}_{1} \\
a^{5} \mathrm{D}_{0}\end{array}$ & $\begin{array}{l}8,307.5 \\
8,095.2 \\
7,927.4 \\
7,810.7 \\
7,750.7\end{array}$ & $\begin{array}{r}212.3 \\
167.8 \\
116.7 \\
60.0\end{array}$ \\
\hline $\begin{array}{l}g^{7} \mathrm{D}_{5} \\
g^{7} \mathrm{D}_{4} \\
g^{7} \mathrm{D}_{3} \\
g^{7} \mathrm{D}_{2} \\
g^{7} \mathrm{D}_{1}\end{array}$ & $\begin{array}{l}47,709.8 \\
47,705.3 \\
47,702.5 \\
47,700.6 \\
47,699.2\end{array}$ & $\begin{array}{l}4.5 \\
2.9 \\
1.8 \\
1.4\end{array}$ & $\begin{array}{l}b^{5} \mathrm{D}_{4} \\
b^{5} \mathrm{D}_{3} \\
b^{5} \mathrm{D}_{2} \\
b^{5} \mathrm{D}_{1} \\
b^{5} \mathrm{D}_{0}\end{array}$ & $\begin{array}{l}24,282.3 \\
24,303.9 \\
24,299.8 \\
24,286.6 \\
24,277.1\end{array}$ & $\begin{array}{r}-21.6 \\
+4.1 \\
13.2 \\
9.5\end{array}$ \\
\hline
\end{tabular}

4 Anales. Soc. Españ. Fis. Quim., 28, p. 611; 1930.

8 Phys. Rov., 33, p. 900; 1920 .

6 Linienspektren und periodisches System der Elemente; Julius Springer, Berlin; 1927. 
TABLE 1.-Terms of $\mathrm{Cr}$ I-Continued

\begin{tabular}{|c|c|c|c|c|c|}
\hline Term & $\nu$ & $\Delta \nu$ & Term & $\nu$ & $\Delta \nu$ \\
\hline $\begin{array}{l}e^{5} \mathrm{D}_{4} \\
e^{5} \mathrm{D}_{3} \\
e^{5} \mathrm{D}_{2} \\
e^{5} \mathrm{D}_{1} \\
e^{5} \mathrm{D}_{0}\end{array}$ & $\begin{array}{c}48,824.3 \\
48,661.4 \\
48,558.5 \\
? \\
?\end{array}$ & $\begin{array}{l}162.9 \\
102.9\end{array}$ & $\begin{array}{l}t^{5} \mathrm{P}_{3}{ }^{\circ} \\
t^{5} \mathrm{P}_{2}^{\circ} \\
t^{5} \mathrm{P}_{1}^{\circ} \\
s^{5} \mathrm{P}_{3}{ }^{\circ}\end{array}$ & $\begin{array}{c}? \\
49,598.0 \\
49,588.8 \\
49,812.3\end{array}$ & $\begin{array}{l}? \\
9.2\end{array}$ \\
\hline $\begin{array}{l}a^{5} \mathrm{G}_{6} \\
a^{5} \mathrm{G}_{5}\end{array}$ & $\begin{array}{l}20,519.3 \\
20,523.8\end{array}$ & -4.5 & $\begin{array}{l}s^{5} \mathrm{P}_{2}{ }^{\circ} \\
s^{5} \mathrm{P}_{1}^{\circ}\end{array}$ & $\begin{array}{c}49,812.3 \\
49,822.5 \\
?\end{array}$ & $\begin{array}{c}-10.2 \\
?\end{array}$ \\
\hline $\begin{array}{l}a^{5} \mathrm{G}_{4} \\
a^{5} \mathrm{G}_{3} \\
a^{5} \mathrm{G}_{2}\end{array}$ & $\begin{array}{l}20,523.6 \\
20,521.0 \\
20,517.5\end{array}$ & $\begin{array}{r}+0.2 \\
2.6 \\
3.5\end{array}$ & $\begin{array}{l}z^{5} \mathrm{D}_{4}^{\circ}{ }^{\circ} \\
z^{5} \mathrm{D}_{3}^{\circ} \\
z^{5} \mathrm{D}_{2}^{\circ} \\
{ }^{5}{ }^{\circ}\end{array}$ & $\begin{array}{l}33,816.0 \\
33,671.4 \\
33,542.0\end{array}$ & $\begin{array}{l}144.6 \\
129.4 \\
118.4\end{array}$ \\
\hline $\begin{array}{l}z^{7} \mathrm{P}_{4}^{\circ} \\
z^{7} \mathrm{P}_{3}^{\circ} \\
{ }^{7} \mathrm{P}^{\circ}\end{array}$ & $\begin{array}{l}23,498.9 \\
23,386.4\end{array}$ & $\begin{array}{r}112.5 \\
81.4\end{array}$ & $\begin{array}{l}z^{5} \mathrm{D}_{1}^{\circ} \\
z^{5} \mathrm{D}_{0}^{\circ}\end{array}$ & $\begin{array}{l}33,423.6 \\
33,337.6\end{array}$ & 86.0 \\
\hline $\begin{array}{l}z^{7} \mathrm{P}_{2}{ }^{\circ} \\
y^{7} \mathrm{P}_{4}{ }^{\circ} \\
y^{7} \mathrm{P}_{3}{ }^{\circ} \\
y^{7} \mathrm{P}_{2}{ }^{\circ}\end{array}$ & $\begin{array}{l}27,935.3 \\
27,820.3 \\
27,728.9\end{array}$ & $\begin{array}{r}115.0 \\
91.4\end{array}$ & $\begin{array}{l}y^{5} \mathrm{D}_{4}{ }^{\circ} \\
y^{5} \mathrm{D}_{3}^{\circ} \\
y^{5} \mathrm{D}_{2}^{\circ} \\
y^{5} \mathrm{D}_{1}^{\circ} \\
y^{5} \mathrm{D}_{0}^{\circ}\end{array}$ & $\begin{array}{l}41,782.2 \\
41,575.2 \\
41,408.9 \\
41,289.2 \\
41,124.7\end{array}$ & $\begin{array}{l}207.0 \\
166.3 \\
119.7 \\
164.5\end{array}$ \\
\hline $\begin{array}{l}x^{7} \mathrm{P}_{4}{ }^{\circ} \\
x^{7} \mathrm{P}_{3}{ }^{\circ} \\
x^{7} \mathrm{P}_{2}{ }^{\circ}\end{array}$ & $\begin{array}{l}42,277.0 \\
42,251.9 \\
42,237.0\end{array}$ & $\begin{array}{l}25.1 \\
14.9\end{array}$ & $\begin{array}{l}x^{5} \mathrm{D}_{4}{ }^{\circ} \\
x^{5} \mathrm{D}_{3}{ }^{\circ} \\
x^{5} \mathrm{D}_{2}{ }^{\circ} \\
x^{5} \mathrm{D}_{1}^{\circ}\end{array}$ & $\begin{array}{l}42,908.4 \\
42,648.4 \\
42,438.8 \\
42,292.8\end{array}$ & $\begin{array}{l}260.0 \\
209.6 \\
146.0\end{array}$ \\
\hline $\begin{array}{l}z^{7} \mathrm{D}_{5}^{\circ} \\
z^{7} \mathrm{D}_{4}^{\circ}\end{array}$ & $\begin{array}{l}27,825.2 \\
27,649.7\end{array}$ & $\begin{array}{l}175.5 \\
149.4\end{array}$ & $x^{5} \mathrm{D}_{0}^{\circ}$ & $\begin{array}{l}42,292.8 \\
42,218.3\end{array}$ & 74.5 \\
\hline $\begin{array}{l}z^{7} \mathrm{D}_{3}{ }^{\circ} \\
z^{7} \mathrm{D}_{2}{ }^{\circ} \\
z^{7} \mathrm{D}_{1}^{\circ}\end{array}$ & $\begin{array}{l}27,500.3 \\
27,382.1 \\
27,300.3\end{array}$ & $\begin{array}{r}149.4 \\
118.2 \\
31.8\end{array}$ & $\begin{array}{l}w^{5} \mathrm{D}_{4}^{\circ} \\
w^{5} \mathrm{D}_{3}^{\circ}{ }^{\circ} \\
w^{5} \mathrm{D}_{2}^{\circ} \\
v^{5} \mathrm{D}^{\circ}\end{array}$ & $\begin{array}{l}47,866.4 \\
47,814.3 \\
47,786.1\end{array}$ & $\begin{array}{l}\text { 52. } 1 \\
\text { 28. } 2 \\
\text { 13. } 7\end{array}$ \\
\hline $\begin{array}{l}z^{7} \mathrm{~F}_{6}^{\circ} \\
z^{7} \mathrm{~F}_{5}^{\circ}\end{array}$ & $\begin{array}{l}25,771.5 \\
25,548.6\end{array}$ & $\begin{array}{l}222.9 \\
1890\end{array}$ & $w^{5} D_{0}^{\circ}$ & $\begin{array}{l}47,772.4 \\
47,788.1\end{array}$ & -15.7 \\
\hline $\begin{array}{l}z^{7} \mathrm{~F}_{4}^{\circ} \\
z^{7} \mathrm{~F}_{3}^{\circ} \\
z^{7} \mathrm{~F}_{2}^{\circ} \\
z^{7} \mathrm{~F}_{1}^{\circ} \\
z^{7} \mathrm{~F}_{0}^{\circ}\end{array}$ & $\begin{array}{l}25,359.6 \\
25,206.0 \\
25,089.2 \\
25,010.7 \\
24,971.1\end{array}$ & $\begin{array}{r}189.0 \\
153.6 \\
116.8 \\
78.5 \\
39.6\end{array}$ & $\begin{array}{l}v^{5} \mathrm{D}_{4}^{\circ}{ }^{\circ} \\
v^{5} \mathrm{D}_{3}^{\circ} \\
v^{5} \mathrm{D}_{2}^{\circ}{ }^{\circ} \\
v^{5} \mathrm{D}_{1}^{\circ} \\
v^{5} \mathrm{D}_{0}^{\circ}\end{array}$ & $\begin{array}{l}50,557.5 \\
50,628.1 \\
50,654.7 \\
50,662.9 \\
50,661.2\end{array}$ & $\begin{array}{r}-70.6 \\
-26.6 \\
-8.2 \\
+1.7\end{array}$ \\
\hline $\begin{array}{l}z^{5} \mathrm{P}_{3}^{\circ} \\
z^{5} \mathrm{P}_{2}^{\circ} \\
z^{5} \mathrm{P}_{1}^{\circ}\end{array}$ & $\begin{array}{l}26,787.3 \\
26,796.1 \\
26,801.8\end{array}$ & $\begin{array}{l}-8.8 \\
-5.7\end{array}$ & $\begin{array}{l}u^{5} \mathrm{D}_{4}^{\circ} \\
u^{5} \mathrm{D}_{3}^{\circ} \\
u^{5} \mathrm{D}_{2}^{\circ}\end{array}$ & $\begin{array}{l}52,064.6 \\
52,031.9 \\
52,012.8\end{array}$ & $\begin{array}{l}\text { 32. } 7 \\
\text { 19. } 1\end{array}$ \\
\hline $\begin{array}{l}y^{5} \mathrm{P}_{3}{ }^{\circ} \\
y^{5} \mathrm{P}_{2}{ }^{\circ} \\
y^{5} \mathrm{P}_{1}{ }^{\circ}\end{array}$ & $\begin{array}{l}29,824.7 \\
29,584.5 \\
29,420.7\end{array}$ & $\begin{array}{l}240.2 \\
163.8\end{array}$ & $\begin{array}{l}u^{5} \mathrm{D}_{1}^{\circ} \\
u^{5} \mathrm{D}_{0}^{\circ} \\
z^{5} \mathrm{~F}_{5}^{\circ}\end{array}$ & $\begin{array}{l}52,003.7 \\
52,000.5 \\
31,280.0\end{array}$ & $\begin{array}{l}9.1 \\
\text { 3. } 2\end{array}$ \\
\hline $\begin{array}{l}x^{5} \mathrm{P}_{3}{ }^{\circ} \\
x^{5} \mathrm{P}_{2}^{\circ} \\
x^{5} \mathrm{P}_{1}^{\circ}\end{array}$ & $\begin{array}{l}41,043.4 \\
40,982.7 \\
40,930.2\end{array}$ & $\begin{array}{l}60.7 \\
52.5\end{array}$ & $\begin{array}{l}z^{5} \mathrm{~F}_{4}{ }^{\circ} \\
z^{5} \mathrm{~F}_{3}^{\circ}{ }^{\circ} \\
z^{5} \mathrm{~F}_{2}^{\circ}{ }^{\circ} \\
z^{5} \mathrm{~F}_{1}{ }^{\circ}\end{array}$ & $\begin{array}{l}31,280 . \\
31,106.2 \\
30,965.2 \\
30,858.5 \\
30,787.1\end{array}$ & $\begin{array}{r}173.8 \\
141.0 \\
106.7 \\
71.4\end{array}$ \\
\hline $\begin{array}{l}w^{5} \mathrm{P}_{3}{ }^{\circ} \\
w^{5} \mathrm{P}_{2}^{\circ} \\
w^{5} \mathrm{P}_{1}^{\circ}\end{array}$ & $\begin{array}{l}44,259.5 \\
44,187.0 \\
44,125.9\end{array}$ & $\begin{array}{l}72.5 \\
61.1\end{array}$ & $\begin{array}{l}y^{5} \mathrm{~F}_{5}^{\circ} \\
y^{5} \mathrm{~F}_{4}^{\circ} \\
y^{5} \mathrm{~F}_{3}^{\circ}\end{array}$ & $\begin{array}{l}41,393.4 \\
41,224.8 \\
41,086.3\end{array}$ & $\begin{array}{l}168.6 \\
138.5 \\
114.7\end{array}$ \\
\hline $\begin{array}{l}v^{5} \mathrm{P}_{3}{ }^{\circ} \\
v^{5} \mathrm{P}_{2}^{\circ} \\
v^{5} \mathrm{P}_{1}^{\circ}\end{array}$ & $\begin{array}{l}45,113.1 \\
44,875.0 \\
44,666.7\end{array}$ & $\begin{array}{l}238.1 \\
208.3\end{array}$ & $\begin{array}{l}y^{5} \mathrm{~F}_{2}{ }^{\circ} \\
y^{5} \mathrm{~F}_{1}^{\circ} \\
x^{5} \mathrm{~F}_{5}{ }^{\circ}\end{array}$ & $\begin{array}{l}40,971.6 \\
40,906.5 \\
45,305.8\end{array}$ & $\begin{array}{r}114.7 \\
65.1\end{array}$ \\
\hline $\begin{array}{l}u^{5} \mathrm{P}_{3}^{\circ}{ }^{\circ} \\
u^{5} \mathrm{P}_{2}^{\circ} \\
u^{5} \mathrm{P}_{1}^{\circ}\end{array}$ & $\begin{array}{l}46,878.5 \\
46,967.6 \\
47,021.9\end{array}$ & $\begin{array}{l}-89.1 \\
-54.3\end{array}$ & $\begin{array}{l}x^{5} \mathrm{~F}_{4}{ }^{\circ} \\
x^{5} \mathrm{~F}_{3}^{\circ}{ }^{\circ} \\
x^{5} \mathrm{~F}_{2}^{\circ} \\
x^{5} \mathrm{~F}_{1}{ }^{\circ}\end{array}$ & $\begin{array}{l}45,285.8 \\
45,255.3 \\
45,224.9 \\
45,201.5\end{array}$ & $\begin{array}{l}20.0 \\
30.5 \\
30.4 \\
23.4\end{array}$ \\
\hline
\end{tabular}

$4754^{\circ}-30-18$ 
TABLE 1.-Terms of $\mathrm{Cr} \mathrm{I}$-Continued

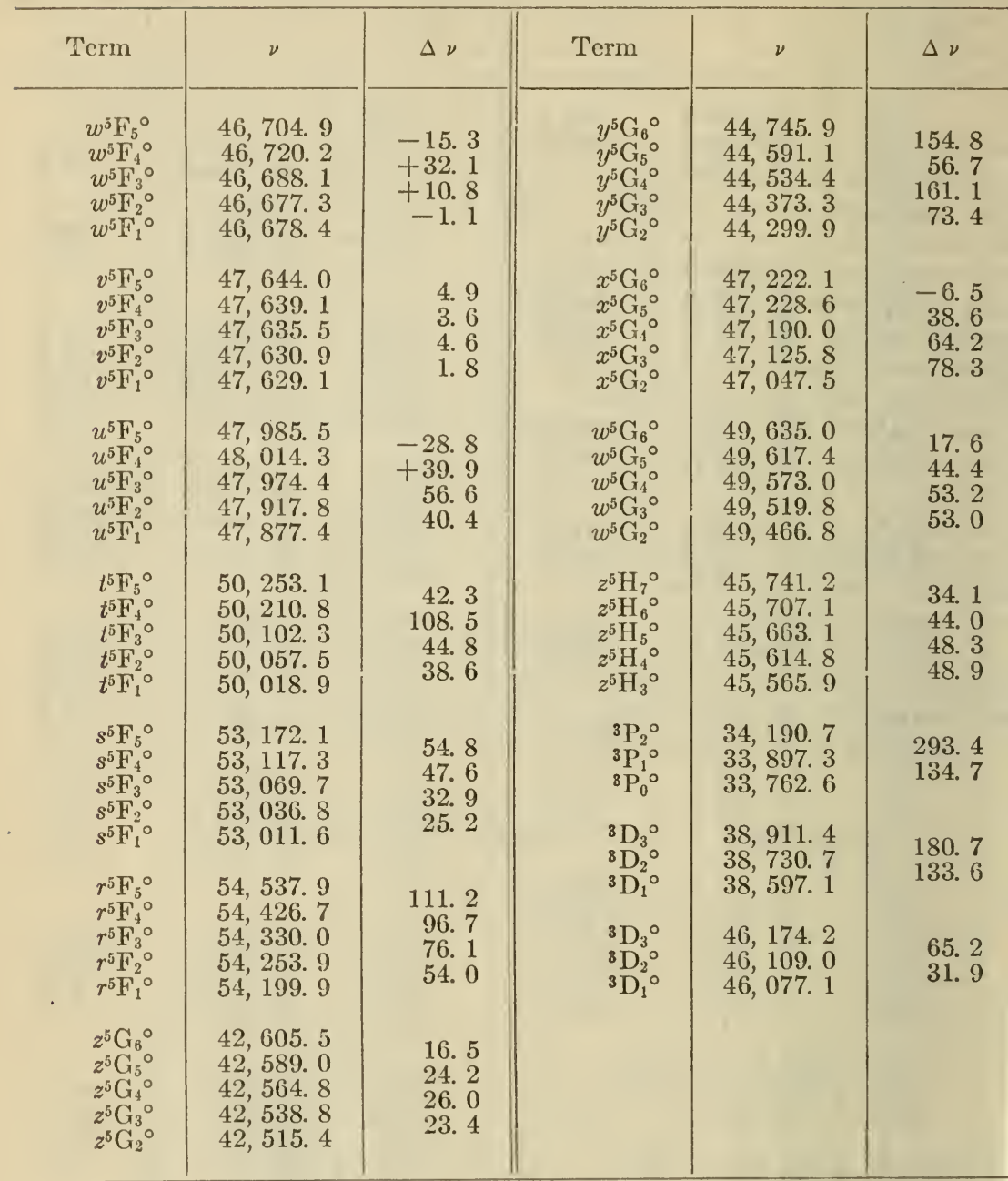

TABLE 2.-T'rms of $\mathrm{Cr} I I$

\begin{tabular}{|c|c|c|c|c|c|}
\hline Term & $\nu$ & $\Delta \nu$ & Term & $\nu$ & $\Delta \nu$ \\
\hline$a^{6} \mathrm{~S}_{216}$ & 0.0 & & $a^{4} \mathrm{D}_{336}$ & $20,025.5$ & 226.0 \\
\hline$a^{6} \mathrm{D}_{4 ! 4}$ & $12,498.3$ & 192.6 & $a^{4} \mathrm{D}_{115}$ & $19,632.9$ & $\begin{array}{l}166.6 \\
103.0\end{array}$ \\
\hline $\begin{array}{l}a^{6} \mathrm{D}_{336} \\
a^{6} \mathrm{D}_{2 ! 3}\end{array}$ & $\begin{array}{l}12,305.7 \\
12,149.7\end{array}$ & 156. 0 & $a^{4} D_{03\}}$ & $19,529.9$ & \\
\hline $\begin{array}{l}a^{6} \mathrm{D}_{13 / 5} \\
a^{6} \mathrm{D}_{013}\end{array}$ & $\begin{array}{l}12,034.3 \\
11,963.5\end{array}$ & $\begin{array}{r}115.4 \\
70.8\end{array}$ & $\begin{array}{l}b^{4} \mathrm{D}_{3 / 4} \\
b^{4} \mathrm{D}_{21 / 6}\end{array}$ & $\begin{array}{l}25,035.4 \\
25,048.4\end{array}$ & $\begin{array}{r}-13.0 \\
+3.9\end{array}$ \\
\hline $\begin{array}{l}a^{4} \mathrm{P}_{21 / 6} \\
a^{4} \mathrm{P}_{13,} \\
a^{4} \mathrm{P}_{016}\end{array}$ & $\begin{array}{l}30,866.1 \\
30,309.1 \\
29,953.8\end{array}$ & $\begin{array}{l}557.0 \\
355.3\end{array}$ & $b^{4} \mathrm{D}_{035}$ & $25,037.3$ & \\
\hline
\end{tabular}


